\title{
Curvas de crescimento em ovinos da raça morada nova criados no estado da Bahia ${ }^{1}$
}

\section{Laaina de Andrade Souza ${ }^{2}$, Paulo Luiz Souza Carneiro², Carlos Henrique Mendes Malhado², Samuel Rezende Paiva ${ }^{3}$, Dirlane Novais Caires ${ }^{2}$, Débora Louise Ferreira Barreto ${ }^{2}$}

\author{
1 Pesquisa financiada pelo Banco do Nordeste, Fapesb, CNPq. \\ 2 Universidade Estadual do Sudoeste da Bahia . \\ ${ }^{3}$ Embrapa-Cenargen.
}

RESUMO - Os objetivos neste estudo foram analisar modelos não-lineares para descrever o crescimento em ovinos da raça Morada Nova criados no estado da Bahia e, após a definição do modelo de melhor ajuste, calcular a taxa de crescimento absoluto e avaliar a influência de fatores ambientais (sexo e tipo de parto) sobre os parâmetros da curva. Utilizaram-se dados de pesagem de 40 ovinos, do nascimento aos 385 dias de idade. Os modelos não-lineares utilizados foram: Brody, Von Bertalanffy, Richards, Logístico e Gompertz. Os modelos Von Bertalanffy, Brody e Richards foram os que apresentaram os melhores ajustes e são equivalentes para explicar o crescimento dos animais. A taxa de crescimento absoluto teve acentuado decréscimo na fase pós-desmama, o que indica a necessidade de se adotar um regime alimentar adequado para acompanhar as exigências de nutrientes dos animais nessa fase. Os efeitos ambientais (sexo e tipo de parto) influenciaram significativamente a taxa a maturidade $(k)$, mas apenas o sexo do animal afetou o peso à maturidade $(A)$. Os animais que nasceram de parto simples tiveram peso maior que o daqueles de parto duplo, e estes apresentaram ganho compensatório. Os machos tiveram maior crescimento que as fêmeas, que, por sua vez, apresentaram maior precocidade. A correlação estimada entre os parâmetros $A$ e $k$ foi negativa $(-0,65)$ para o modelo Von Bertalanffy e $(-0,71)$ para os modelos Brody e Richards, e isso indica que os animais mais precoces possuem menor probabilidade de atingir pesos elevados à idade adulta.

Palavras-chave: efeito ambiental, modelos não-lineares, peso à maturidade

\section{Growth curves in morada nova sheep raised in the state of Bahia}

\begin{abstract}
The objective of the present study was to evaluate non-linear models to describe Morada Nova sheep herds raised in the State of Bahia and, after defining the best suiting model, calculate the rate of absolute growth and evaluate the influence of environmental factors (sex and type of parturition) on the curve parameters. Weighting data from 40 sheep from birth up to 385 days of age were used. The non-linear models used were: Brody, Von Bertalanffy, Richards, Logistic and Gompertz. Von Bertalanffy, Brody and Richards models presented the best adjustments and were equivalent to explain the growth of the analyzed animals. A remarkable drop in the absolute growth rate was observed after weaning, suggesting that a proper diet should be implemented to supply the nutritional demands of the animals at this stage. The environmental effects (sex and type of parturition) have significantly influenced the maturity rate ( $k$ ), but only the sex of the lamb affected weight at maturity $(A)$. Animals born from single parturition proved to be heavier than twins which, in turn, presented compensatory gain. The growth of males was higher than of females and the latter presented more precocity than males. Estimated correlation between parameters $A$ and $k$ was negative $(-0.65)$ for the Von Bertalanffy model and for the Brody and Richards models $(-0.71)$ indicating that the most precocious animals are less likely to reach elevated weights when adults.
\end{abstract}

Key Words: environmental effect, non-linear models, weight at maturity

\section{Introdução}

A Região Nordeste possui 9.286.258 milhões de ovinos, correspondente a 57\% dos rebanhos do País. O estado da Bahia concentra os maiores plantéis dessa espécie, com 3.096.155 milhões de ovinos (IBGE, 2008), sobressaindo como um estado com vocação para a exploração de pequenos ruminantes domésticos, principalmente devido à sua grande extensão territorial e mão-de-obra de baixo custo.

Os ovinos Morada Nova são uma das principais raças locais de ovinos deslanados do Nordeste do Brasil, sendo mantidos em pequenos efetivos populacionais no estado da Bahia. São criados para carne e pele, sendo esta altamente apreciada no mercado internacional. 
Com a expansão do mercado da carne ovina no Brasil, são necessárias pesquisas para determinação do peso de abate dos grupos genéticos mais adequados às condições locais, visando máxima eficiência produtiva e econômica para atender às exigências dos consumidores (FurushoGarcia et al., 2004). O mercado sinaliza para o consumo de carne de animais jovens, abatidos com até seis meses de idade, mas a predominância é o abate de animais velhos e com carcaças de baixa qualidade e rendimento (Leite, 2005).

Vários estudos já foram realizados com curvas de crescimento em ovinos (McManus et al., 2003; Guedes et al., 2004; Sarmento et al., 2006; Lôbo et al., 2006; Carneiro et al., 2007; Malhado et al., 2008a; Malhado et al., 2009) e caprinos (Freitas, 2005; Malhado et al., 2008b; Oliveira et al., 2009; Carneiro et al., 2009) entretanto não existe nenhum estudo específico com ovinos da raça Morada Nova.

Muitas das raças locais, como a Morada Nova, estão hoje ameaçadas de extinção. A conservação e ampliação dos rebanhos dessas raças passam, necessariamente, pelo aproveitamento desses recursos genéticos em sistemas de produção. Além disso, essas raças podem ser importantes como fontes de genes para programas de melhoramento animal em um futuro próximo. Assim, estudos para avaliar o desempenho de animais adaptados às condições locais para características de importância econômica são imprescindíveis para salvaguardar e, acima de tudo, utilizar esse recurso genético em benefício da sociedade.

Os objetivos neste trabalho foram analisar modelos não-lineares para descrever o crescimento em ovinos da raça Morada Nova criados no estado da Bahia e, após a definição do modelo de melhor ajuste, calcular a taxa de crescimento absoluto e avaliar a influência dos fatores ambientais (tipo de parto e sexo) sobre os parâmetros ( $A$ e $k$ ) da curva.

\section{Material e Métodos}

Foram coletados dados na Estação Experimental de Jaguaquara, pertencente à Empresa Baiana de Desenvolvimento Agrícola S.A. - EBDA, localizada no município de Jaguaquara-BA, microrregião administrativa de Jequié-BA, no período de outubro de 2006 a novembro de 2007. A precipitação neste período foi de $1.171 \mathrm{~mm}$. Foram utilizados 40 animais, sendo pesados quinzenalmente do nascimento a aproximadamente 385 dias de idade. As crias acompanharam as matrizes nas pastagens até os 120 dias, quando foram desmamadas. Após o desmame, foram mantidos em sistema de pastejo durante o dia, em áreas cultivadas com capim Panicum maximum e Brachiaria decumbens, com cobertura de $30 \%$ e $70 \%$, respectivamente, com sal mineral comercial a disposição, e recolhidos no final da tarde para o aprisco.

Foram ajustados os modelos Brody, Von Bertalanffy, Richards, Logístico e Gompertz (Tabela 1) para se estimar o crescimento do animal e os parâmetros da curva, em que $Y$ é o peso corporal à idade $t ; A$, o peso assintótico, sendo esse parâmetro interpretado como peso à idade adulta; $B$, uma constante de integração, relacionada aos pesos iniciais do animal. $\mathrm{O}$ valor de $B$ é estabelecido pelos valores iniciais de $Y$ e $t$. $O k$ é interpretado como taxa de maturação, que é a mudança de peso em relação ao peso e à maturidade, ou seja, indicador da velocidade com que o animal se aproxima do seu tamanho adulto. O parâmetro $m$ é que dá forma à curva e, conseqüentemente, determina em que proporção do valor assintótico $(A)$ ocorre o ponto de inflexão da curva.

No caso dos modelos não-lineares, não é possível resolver o sistema de equações formado diretamente, como ocorre no caso linear, uma vez que a resolução do sistema é dependente dos próprios parâmetros a serem estimados. Dessa forma, usa-se um processo iterativo para obtenção das estimativas dos parâmetros, o qual começa com valores iniciais, atribuídos aos próprios parâmetros a serem estimados. Calcula-se, então, a soma de quadrado do erro e, a cada passo, obtém-se um conjunto de estimativas atualizadas até o procedimento convergir para um vetor final de estimativas, obtendo-se a soma mínima de quadrados do erro (Sarmento et al., 2006). Os parâmetros dos modelos foram estimados pelo método de Gauss Newton modificado por meio do procedimento NLIN do SAS (2003), usando o critério de convergência de $10^{-8}$.

Os critérios utilizados para seleção do modelo que melhor descreveu a curva de crescimento foram: quadrado médio do resíduo (QMR) - calculado dividindo-se a soma de quadrados do resíduo, pelo número de observações, que é o estimador de máxima verossimilhança da variância residual, para que se pudesse comparar o QMR dos diferentes modelos, visto que possuem números diferentes de parâmetros a serem estimados; coeficiente de determinação $\left(\mathrm{R}^{2}\right)$ - calculado como o quadrado da correlação entre os pesos observados e estimados, através de uma análise de regressão linear considerando o peso predito pelo modelo não linear como variável dependente e o peso observado do

Tabela 1 - Forma geral dos modelos não-lineares

\begin{tabular}{lc}
\hline Modelo & Forma geral \\
\hline Brody & $y=A\left(1-B e^{-k t}\right)+\varepsilon$ \\
Von Bertalanffy & $y=A\left(1-B e^{-k t}\right)^{3}+\varepsilon$ \\
Richards & $y=A\left(1-B e^{-k t}\right)^{m}+\varepsilon$ \\
Logístico & $y=A\left(1+B e^{-k t}\right)^{-m}+\varepsilon$ \\
Gompertz & $y=A e^{-B e^{-k t}+\varepsilon}$ \\
\hline
\end{tabular}


animal como variável independente; percentual de convergência (C\%) - considerando a existência ou não de convergência; análise gráfica das curvas estimadas e observadas; e desvio médio absoluto dos resíduos (DMA) - estatística proposta por Sarmento et al. (2006) para avaliar a qualidade de ajuste, calculado como:

DMA $=\frac{\sum_{i=1}^{n}\left|Y_{i}-\hat{Y}_{i}\right|}{n}$, sendo que o $Y_{i}$ é o valor observado, $\hat{Y}_{i}$ o valor estimado e $n$ o tamanho da amostra. Quanto menor o valor do DMA, melhor o ajuste.

Depois de selecionado o modelo, calculou-se a taxa de crescimento absoluta (TCA), obtida a partir da primeira derivada do modelo ajustado, em relação ao tempo $(\partial Y / \partial t)$. A TCA é, na realidade, o ganho de peso obtido por unidade de tempo e, como, nesse caso, o tempo está em dia, representa o ganho de peso médio diário, estimado ao longo da trajetória de crescimento.

Na etapa seguinte, foi avaliada a influência do sexo (macho e fêmea) e tipo de parto (simples e duplo) sobre os parâmetros da curva do modelo selecionado, através do procedimento GLM do software SAS (2003), bem como as correlações de Pearson entre os parâmetros foram estimados.

Depois de constatada a convergência de todos os modelos, confeccionou-se um arquivo de dados contendo a identificação do modelo e os valores correspondentes a cada avaliador (quadrado médio do resíduo-QMR, coeficiente de determinação - $\mathrm{R}^{2}$, percentual de convergência - \%C e desvio médio absoluto-DMA). Esses dados foram submetidos à análise de agrupamento no PROC CLUSTER, do software SAS (2003), pelo método centroide, objetivando reunir os modelos de forma que exista homogeneidade dentro e heterogeneidade entre os grupos de acordo com os valores dos avaliadores.

\section{Resultados e Discussão}

Em estudos de curvas de crescimento, não só o aspecto estatístico deve ser levado em consideração na identificação dos melhores modelos, mas também é necessário avaliar sob o ponto de vista biológico as estimativas dos parâmetros produzidas por eles (Mendes et al., 2008).

O parâmetro A (Tabela 2) representa a estimativa do peso assintótico, que é interpretado como peso adulto. Esse peso não é o máximo que o animal atinge, e sim o peso médio à maturidade livre das variações sazonais (Brown et al., 1976). As estimativas do parâmetro $A$ na raça Morada Nova foi maior para Brody $(28,96)$, Richards $(28,96)$ e Bertalanffy $(28,07)$ seguidos dos modelos Gompertz $(26,82)$ e Logístico $(26,01)$. Esses resultados foram menores, quando comparados a ovinos mestiços Santa Inês $\times$ Texel criados no estado da Bahia. Neste estudo, realizado por Malhado et al. (2008a), o parâmetro $A$ apresentou maior valor para os modelos Brody e Richards $(37,7)$. Contudo, o parâmetro A na raça Morada Nova foi maior se comparado ao do estudo de Sarmento et al. (2006) com ovinos Santa Inês puros criados no estado da Paraíba, em que obtiveram os seguintes resultados: modelos Brody $(27,4)$, Bertalanffy $(24,8)$, Richards (24,5), Gompertz (24,2) e Logístico $(23,2)$.

O parâmetro $k$ (Tabela 2) representa a taxa de maturidade do animal e indica a velocidade de crescimento para atingir o peso assintótico. Segundo Malhado et al. (2009), os animais com altos valores de $k$ apresentam maturidade precoce, em comparação a animais de valores menores de $k$ e de peso inicial similar. Devido à pequena variação no peso ao nascimento, a variação entre os valores de $k$ representa, com boa precisão, as variações na velocidade relativa que o animal cresce. Com relação a esse parâmetro, a estimativa do modelo Richards $(0,217)$ foi superior à dos modelos Logístico $(0,013)$, Gompertz $(0,011)$, Von Bertalanffy $(0,009)$ e Brody $(0,006)$. Malhado et al. (2008a), obtiveram resultados semelhantes para $k$ no modelo Richards $(0,202)$ que apresentou valor superior aos modelos Logístico $(0,014)$, Gompertz $(0,012)$, Bertalanffy $(0,010)$ e Brody $(0,006)$. Sarmento et al. (2006) obtiveram maior $k$ para o modelo Logístico $(0,028)$ e menor para o Brody $(0,009)$. Isso representa uma maior velocidade de crescimento dos animais da raça

Tabela 2 - Estimativas dos parâmetros, quadrado médio do resíduo, coeficiente de determinação, percentual de convergência e desvio médio absoluto de acordo com os modelos estudados

\begin{tabular}{|c|c|c|c|c|c|}
\hline \multirow[t]{2}{*}{ Parâmetro } & \multicolumn{5}{|c|}{ Modelo } \\
\hline & Bertalanffy & Brody & Gompertz & Logístico & Richards \\
\hline A & 28,07 & 28,96 & 26,82 & 26,01 & 28,96 \\
\hline$B$ & 0,47 & 0,88 & 1,84 & - & 0,88 \\
\hline K & 0,009 & 0,006 & 0,011 & 0,013 & 0,217 \\
\hline$m$ & - & - & - & 2,53 & 0,03 \\
\hline Quadrado médio do resíduo & 2,87 & 2,67 & 3,00 & 3,13 & 2,67 \\
\hline Coeficiente de determinação & 0,944 & 0,949 & 0,941 & 0,938 & 0,949 \\
\hline Percentual de convergência & 93,94 & 93,94 & 96,97 & 96,97 & 93,94 \\
\hline Desvio médio absoluto & 1,175 & 1,097 & 1,224 & 1,271 & 1,097 \\
\hline
\end{tabular}


Santa Inês puros em relação aos da raça Morada Nova e mestiços Santa Inês $\times$ Texel.

Com base no coeficiente de determinação $\left(\mathrm{R}^{2}\right)$ (Tabela 2), os modelos Von Bertalanffy, Brody e Richards tiveram melhores ajustes que os demais, com valores próximos a 95\%. Esses modelos apresentaram ainda menor desvio médio absoluto (DMA), menor quadrado médio do resíduo (QMR), e percentual de convergência superior a 90\%, sendo equivalentes para explicar o crescimento dos animais e considerados os melhores modelos.

Esse fato foi confirmado pela análise de agrupamento, a qual indicou a formação de dois grupos (Figura 1), um com os modelos Von Bertalanffy, Brody e Richards (Figura 2) e outro com os modelos Gompertz e Logístico (Figura 3). Entre os modelos selecionados (Von Bertalanffy, Brody e Richards), os mais similares, com relação aos avaliadores, foram Brody e Richards, sendo que o Von Betalanffy apresentou pequenas diferenças em relação a estes. Considerando as curvas desses modelos em relação à curva média observada (Figura 2), todos apresentaram comportamento semelhante com bom ajuste até os 150 dias e superestimação dos pesos a partir dessa idade, o que aconteceu também com os modelos não-selecionados (Gompertz e Logístico).

Freitas (2005) estudando a raça Santa Inês verificou que o modelo Von Bertalanffy, que é do tipo sigmoide e que o parâmetro " $A$ ” fornece indicação do peso à maturidade, possibilitou uma curva adequada, demonstrando inclusive o momento de estabilização do crescimento. Ainda na raça Santa Inês, Sarmento et al. (2006) sugerem o modelo Gompertz para descrição da curva de crescimento nesta espécie, uma vez que este modelo apresentou ajuste médio superior aos demais modelos estudados. Malhado et al. (2009), estudando ovinos cruzados Dorper com as raças Morada Nova, Rabo Largo e Santa Inês, escolheram o modelo Logístico, pois este apresentou pequena superioridade em relação ao modelo Gompertz. McManaus et al. (2003) trabalharam com ovinos da raça Bergamácia e recomendaram o modelo Logístico para o ajuste da curva de crescimento, pois, em termos práticos, a curva Logística possui menos parâmetros que a curva de Richards e apresentou maior correlação com os pesos vivo dos animais avaliados.

O modelo Von Bertalanffy foi escolhido para descrever a taxa de crescimento absoluto (TCA) dos animais. A taxa de crescimento absoluto (TCA) foi obtida pela primeira derivada do modelo Von Bertalanffy em função do tempo (Figura 4). A TCA foi crescente até atingir o máximo, aproximadamente $116 \mathrm{~g}$ aos 38 dias. Os animais começaram a ganhar pouco peso após os 120 dias de idade, quando foram desmamados.

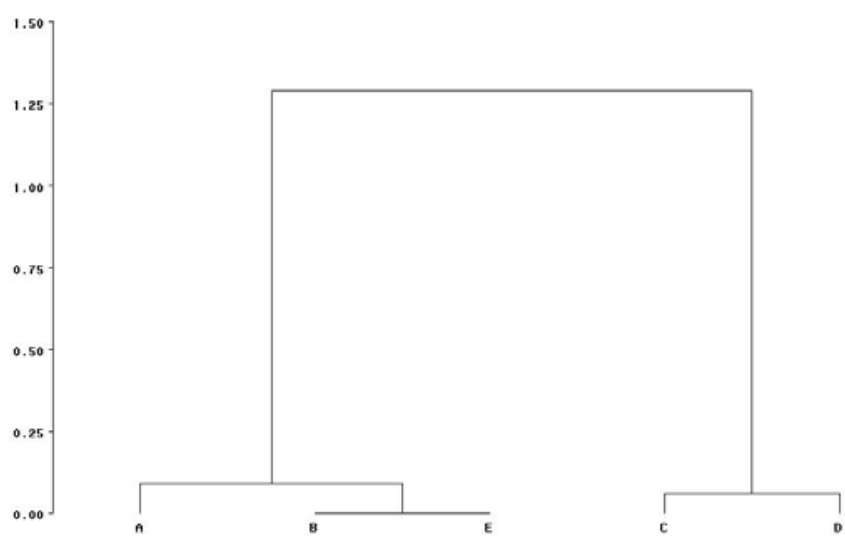

Figura 1 - Dendrograma resultante da análise de agrupamento fundamentada nos avaliadores de qualidade para os modelos Von Bertalanffy (A), Brody (B), Gompertz (C), Logístico (D) e Richards (E).

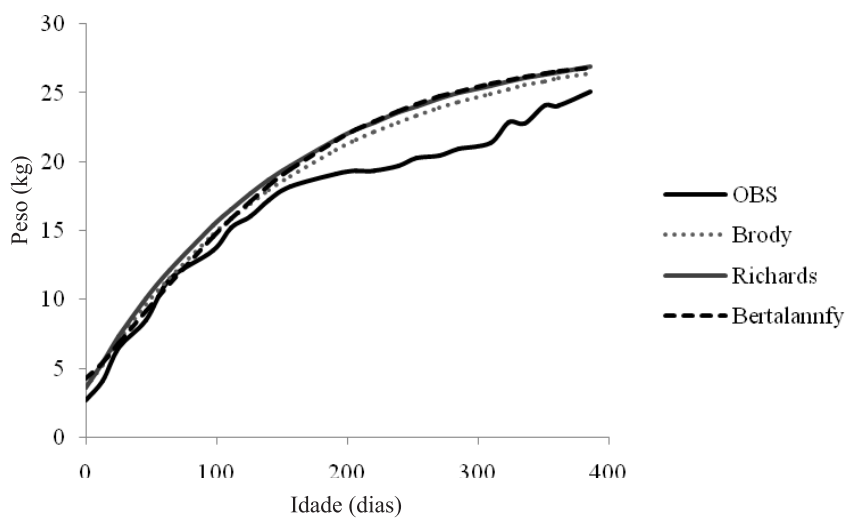

Figura 2 - Peso em função da idade, obtidas pelos modelos Brody, Richards, Bertalanffy e observados em ovinos da raça Morada Nova.

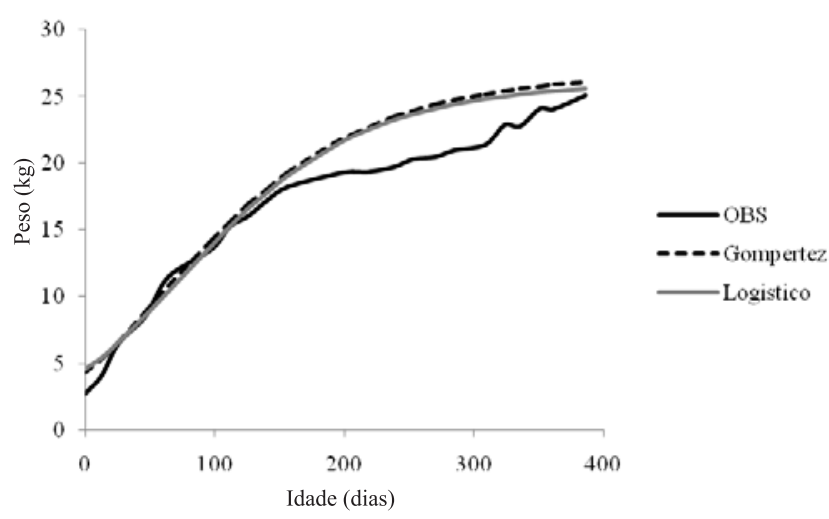

Figura 3 - Peso em função da idade, ajustado pelos modelos Gompertz, Logístico e observados em ovinos da raça Morada Nova. 


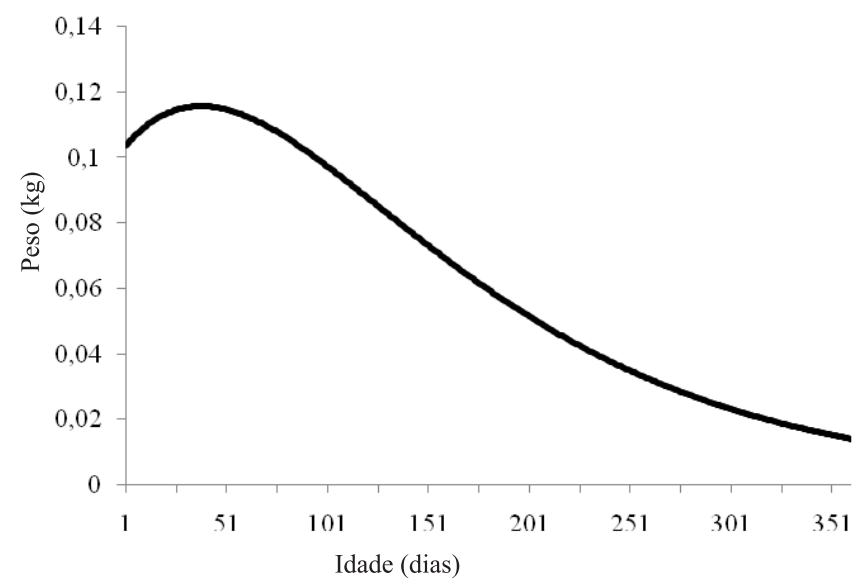

Figura 4 - Taxa de crescimento absoluto estimado pela função Bertalanffy em ovinos da raça Morada Nova.

O ponto de inflexão, ou seja, o ponto em que a função muda de crescente para decrescente, é importante para auxiliar os produtores em programas alimentares específicos e na definição da melhor idade ao abate. Assim, estratégias nutricionais para a fase pós-desmama podem ser implementadas, de forma a minimizar os efeitos da mudança de dieta nesta fase, amenizando o acentuado decréscimo da TCA na pós-desmama e estabelecendo a idade de abate com melhor custo-benefício.

Sarmento et al. (2006) frisaram que o decréscimo da TCA pode ser resultado de manejo inadequado para acompanhar a maior demanda de nutriente à medida que o animal cresce.

Os efeitos ambientais de sexo e o tipo de parto influenciaram significativamente $(\mathrm{P}<0,05)$ a taxa de maturidade. As médias de $k$ foram 0,006 e 0,011 para machos e fêmeas, respectivamente, e 0,011 e 0,007 na mesma ordem, para parto simples e duplo.

Apenas o efeito de sexo influenciou significativamente $(\mathrm{P}<0,05)$ o peso a maturidade $A$, as médias foram 34,91 e $22,43 \mathrm{~kg}$ para machos e fêmeas, respectivamente. Malhado et al. (2008a) encontraram resultados diferentes, em que o sexo do cordeiro não influenciou significativamente $(\mathrm{P}>0,05)$ os parâmetros $(A, k, m)$ da função Logística. Contudo, Bathaei \& Leroy (1996), McManus et al. (2003) e Sarmento et al. (2006) observaram que o sexo do animal influenciou de maneira expressiva os parâmetros $A$ e $k$. Malhado et al. (2008a) analisando o efeito do tipo de parto (simples ou duplo), encontraram resultados significativos $(\mathrm{P}<0,05)$ para o parâmetro $m$. Esse parâmetro define a formada curva no modelo e, conseqüentemente, determina em que proporção do valor assintótico $(A)$ ocorre o ponto de inflexão da curva.

A correlação entre as estimativas dos parâmetros $A$ e $k$ foi significativa $(\mathrm{P}<0,05)$ e igual a $(-0,65)$ para o modelo Von Bertalanffy e (-0,71) para os modelos Brody e Richards corroborando aos achados de McManus et al. (2003), Sarmento et al. (2006) e Malhado et al. (2008 a, b).

McManus et al. (2003) salientam que a relação biológica mais importante para uma curva está entre os parâmetros $A$ e $k$. A correlação negativa existente entre estes parâmetros indica que animais com menores taxas de crescimento atingem maiores pesos à maturidade.

\section{Conclusões}

Apesar de representar pequeno núcleo sob conservação no estado da Bahia, os ovinos da raça Morada Nova apresentam crescimento próximo ao de outros grupos genéticos que vem sendo utilizados em sistemas produtivos, o que sugere a necessidade de ampliação do rebanho e avaliações mais detalhadas do desempenho dessa raça. Os modelos Von Bertalanffy, Brody e Richards apresentam os melhores ajustes e são equivalentes na descrição do crescimento em ovinos da raça Morada Nova criados no Estado da Bahia. A taxa de crescimento observada indica que estratégias nutricionais devem ser implantadas com o objetivo de incrementar o ganho de peso na fase pósdesmama.

\section{Agradecimentos}

À Empresa Baiana de Desenvolvimento Agrícola S.A. - EBDA pela concessão dos dados para o desenvolvimento deste trabalho.

\section{Referências}

BATHAEI, S.S.; LEROY, P.L. Growth and mature weight of Mehraban Iranian fat-tailed sheep. Small Ruminant Research, v.22, p.155-162, 1996

BERTALANFFY, L.V. Quantitative laws in metabolism and growth. The Quarterly Review of Biology, v.32, p.217-230, 1957.

BRODY, S. Bioenergetics and growth. New York: Reinhold Publication, 1945. 1023p.

BROW, J.E.; FITZHUGH JUNIOR, H.A.; CARTWRIGHT, T.C.A. A comparison of nonlinear models for describing weigt-age relationships in cattle. Journal of Animal Science, v.42, p. 810-818, 1976.

CARNEIRO, P.L.S.; MALHADO, C.H.M.; SOUZA JÚNIOR, A.A.O. et al. Desenvolvimento ponderal e diversidade fenotípica entre cruzamentos de ovinos Dorper com raças locais. Pesquisa Agropecuária Brasileira, v.42, p.991-998, 2007.

CARNEIRO, P.L.S.; MALHADO, C.H.M.; AFFONSO, P.R.A.M. et al. Curva de crescimento em caprinos, da raça Mambrina, criados na caatinga. Revista Brasileira de Saúde Produção Animal, v.10, p.536-545, 2009

FREITAS, A.R. Curvas de crescimento na produção animal. Revista Brasileira de Zootecnia, v.34, n.3, p.786-795, 2005.

FURUSHO-GARCIA, I.F.; PEREZ, J.R.O.; BONAGURIO, S. et al. Estudo dos cortes da carcaça de cordeiros Santa Inês Puros e 
Cruzas Santa Inês com Texel, Ile de France e Bergamácia. Revista Brasileira de Zootecnia, v.33, n.2, p.453-462, 2004. GUEDES, M.H.P.; MUNIZ, J.A.; PEREZ, J.R.O. et al. Estudo das curvas de crescimento de cordeiros das raças Santa Inês e Bergamácia considerando heterogeneidade de variâncias. Ciência Agrotécnica, v.28, p.381-388, 2004.

INSTITUTO BRASILEIRO DE GEOGRAFIA E ESTATÍSTICA IBGE. Pesquisa Pecuária Municipal. Rebanho ovino brasileiro: efetivo por unidade da federação 2008. Disponível em: <http:// www.sidra.ibge.gov.br>. Acesso em: 21 ago. 2009.

KHATTREE, R.; NAIK, D.N. Multivariate data reduction and discrimination with SAS software. New York: BBU Press and John Wiley Sons Inc., 2000. 574p.

LAIRD, A.K. Dynamics of relative growth. Growth, v.29, p.249-263, 1965.

LEITE, E.R. A cadeia produtiva da ovinocultura e da caprinocultura de corte In: Do campus para o campo: tecnologias para produção de ovinos e caprinos. Fortaleza: Gráfica Nacional, 2005. p.21-32.

LÔBO, R.N.B.; VILLELA, L.C.V.; LÔBO, A.M.B.O. et al. Parâmetros genéticos de características estimadas da curva de crescimento de ovinos da raça Santa Inês. Revista Brasileira de Zootecnia, v.35, p.1012-1019, 2006 (supl.).

MALHADO, C.H.M.; CARNEIRO, P.L.S.; SANTOS, P.F. et al. Curva de crescimento em ovinos mestiços Santa Inês x Texel criados no Sudoeste do Estado da Bahia. Revista Brasileira de Saúde Produção Animal, v.9, n.2, p.210-218, 2008a.

MALHADO, C.H.M.; CARNEIRO, P.L.S.; CRUZ, J.F. et al. Curvas de crescimento para caprinos da raça Anglo-Nubiana criados na caatinga: rebanho de elite e comercial. Revista Brasileira de Saúde Produção Animal, v.9, n.4, p.667-671, 2008b.

MALHADO, C.H.M.; CARNEIRO, P.L.S.; MELLO, P.R.A.M. et al. Growth curves in Dorper sheep crossed with the local Brazilian breeds, Morada Nova, Rabo Largo, and Santa Inês. Small Ruminant Research, v.84, p.16-21, 2009.

McMANUS, C.; EVANGELISTA, C.; FERNANDES, L.A.C. et al. Curvas de crescimento de ovinos Bergamácia criados no Distrito Federal. Revista Brasileira de Zootecnia, v.32, p.1207-1212, 2003.

MENDES, P.N. et al. Modelo logístico difásico no estudo do crescimento de fêmeas da raça Hereford. Ciência Rural, v.38, n.7, p.1984-1990, 2008.

NELDER, J.A. The fitting of a generalization of the logistic curve. Biometrics, v.17, p.89-110, 1961.

OLIVEIRA, D.F.; CRUZ, J.F.; CARNEIRO, P.L.S. et al. Desenvolvimento ponderal e características de crescimento de caprinos da raça Anglonubiana criados em sistema semi-intensivo. Revista Brasileira de Saúde Produção Animal, v.10, p.256-265, 2009.

RICHARDS, F.J. A flexible growth functions for empirical use. Journal of Experimental Botany, v.10, p.290-300, 1959.

SARMENTO, J.LR.; REZAZZI, A.J.; SOUZA, W.H. et al. Estudo da curva de crescimento de ovinos Santa Inês. Revista Brasileira de Zootecnia, v.35, p.435-442, 2006.

STATISTICAL ANALYSIS SYSTEM - SAS. User's guide. Version 9.1 ed. Cary: SAS Institute, 2003. (CD-ROM). 